\title{
Identification of DNA hypermethylation of SOX9 in association with bladder cancer progression using CpG microarrays
}

\author{
A Aleman', L Adrien², L Lopez-Serra ${ }^{3}$, C Cordon-Cardo ${ }^{4}$, M Esteller $^{3}$, TJ Belbin $^{2}$ and M Sanchez-Carbayo ${ }^{*, I}$ \\ 'Tumor Markers Group, Molecular Pathology Program, Spanish National Cancer Center, Madrid, Spain; ' 2Department of Pathology, Albert Einstein College \\ of Medicine, Bronx, NY, USA; ${ }^{3}$ Epigenetics Group, Molecular Pathology Program, Spanish National Cancer Center, Madrid, Spain; ${ }^{4}$ Division of Molecular \\ Pathology, Memorial Sloan-Kettering Cancer Center (MSKCC), New York, NY, USA
}

\begin{abstract}
CpG island arrays represent a high-throughput epigenomic discovery platform to identify global disease-specific promoter hypermethylation candidates along bladder cancer progression. DNA obtained from 10 pairs of invasive bladder tumours were profiled vs their respective normal urothelium using differential methylation hybridisation on custom-made CpG arrays $(n=12288$ clones). Promoter hypermethylation of 84 clones was simultaneously shown in at least $70 \%$ of the tumours. SOX 9 was selected for further validation by bisulphite genomic sequencing and methylation-specific polymerase chain reaction in bladder cancer cells $(n=\mid \mathrm{I})$ and primary bladder tumours $(n=\mid \mathrm{O} \mathrm{I})$. Hypermethylation was observed in bladder cancer cells and associated with lack of gene expression, being restored in vitro by a demethylating agent. In primary bladder tumours, SOX9 hypermethylation was present in $56.4 \%$ of the cases. Moreover, SOX9 hypermethylation was significantly associated with tumour grade and overall survival. Thus, this high-throughput epigenomic strategy has served to identify novel hypermethylated candidates in bladder cancer. In vitro analyses supported the role of methylation in silencing SOX9 gene. The association of SOX9 hypermethylation with tumour progression and clinical outcome suggests its relevant clinical implications at stratifying patients affected with bladder cancer.
\end{abstract}

British Journal of Cancer (2008) 98, 466-473. doi:I0.1038/sj.bjc.6604|43 www.bjcancer.com

Published online 18 December 2007

(c) 2008 Cancer Research UK

Keywords: bladder cancer; CpG arrays; methylation

\begin{abstract}
Epigenetic changes and genetic mutations contribute significantly to the onset of human malignancies (Jones and Baylin, 2002; Feinberg et al, 2006). The most common epigenetic event in the human genome is the addition of methyl groups to the carbon-5 position of cytosine nucleotides (Costello et al, 2000; Esteller et al, 2001; Esteller, 2002; Jones and Baylin, 2002; Feinberg et al, 2006). CpG islands are present in one-half of the human genes, and typically overlap with promoters and first exons of genes (Costello et al, 2000; Esteller et al, 2001; Esteller, 2002; Jones and Baylin, 2002; Feinberg et al, 2006). Hypermethylation of CpG islands is frequently associated with inappropriate transcriptional silencing of critical genes including tumour suppressors (Costello et al, 2000; Esteller et al, 2001; Esteller, 2002; Jones and Baylin, 2002; Feinberg et al, 2006). Transcriptional inactivation by CpG island promoter hypermethylation is a well-established mechanism for gene silencing in bladder cancer (Cordon-Cardo et al, 2000; Muto et al, 2000; Lee et al, 2001; Markl et al, 2001; Stoehr et al, 2004; Catto et al, 2005; Chapman et al, 2005; Kim et al, 2005; Marsit et al, 2005; Wolff et al, 2005; Urakami et al, 2006). Genes reported to be epigenetically inactivated by hypermethylation in bladder cancer include $p 16^{I N K 4 a}$, CDKN2A, RUNX3 or RASSF1, among others (Cordon-Cardo et al, 2000; Muto et al, 2000; Lee et al, 2001; Markl et al, 2001; Stoehr et al,
\end{abstract}

*Correspondence: M Sanchez-Carbayo, Tumor Markers Group, 208A, Centro Nacional de Investigaciones Oncologicas, Melchor Fernandez Almagro 3, Madrid E-28029, Spain.

E-mail: mscarbayo@cnio.es

Received 22 October 2007; accepted 19 November 2007; published online 18 December 2007
2004; Catto et al, 2005; Chapman et al, 2005; Kim et al, 2005; Marsit et al, 2005; Wolff et al, 2005; Urakami et al, 2006).

$\mathrm{CpG}$ island arrays represent a high-throughput technology for the discovery of $\mathrm{CpG}$ island loci frequently hypermethylated during disease progression (Yan et al, 2000; Ching et al, 2005). Gene profiling analyses of cancer cells after exposure to demethylating agents may also uncover critical genes susceptible to be epigenetically silenced (Liang et al, 2002). Using a high-throughput epigenomic approach with $\mathrm{CpG}$ arrays, we identify novel candidates such as SOX9, presenting CpG island promoter hypermethylation in bladder cancer. To the best of our knowledge, SOX 9 had not been reported to be epigenetically modified, nor differentially expressed in bladder malignancies. In bladder cancer cell lines, the low expression of SOX 9 was restored by a demethylating agent in methylated cancer cells. Furthermore, SOX9 promoter hypermethylation was frequently found in bladder tumours. Moreover, SOX9 methylation was associated with tumour grade and overall survival of bladder cancer patients. These are relevant findings not only as they relate to cancer progression but also for their utility in the clinical management of patients affected by uroepithelial neoplasias.

\section{METHODS}

\section{Tumour samples, cell lines, genomic DNA and RNA extraction}

Following IRB-approved protocols at MSKCC, primary bladder tumours and paired normal urothelium were frozen in liquid 
nitrogen immediately after resection and stored at $-80^{\circ} \mathrm{C}$ until processing. Bladder tissues embedded in OCT were macrodissected to ensure a minimum of $75 \%$ of tumour or normal urothelium counterpart cell subpopulations (Sanchez-Carbayo et al, 2006). Genomic DNA was extracted using a non-organic method (Oncor, Gaithersburg, MD, USA). Total RNA was isolated in two steps using TRIzol (Life Technologies, Carlsbad, CA, USA), followed by RNeasy purification (Qiagen, Valencia, CA, USA). DNA and RNA quality was evaluated based on 260/280 ratios of absorbances and the integrity was also checked by gel electrophoresis analysis using the Agilent 2100 Bioanalyzer (Agilent Technologies, Palo Alto, CA, USA). For CpG methylation arrays, invasive bladder tumours $(n=10)$ were compared to their paired normal urothelium. An independent series of 101 bladder tumours (non-muscle invasive $(n=56): \mathrm{pTa}(n=24), \mathrm{pT} 1(n=32)$; and muscle invasive $(n=45)$ : pT2 $(n=16)$, pT3 $(n=25)$, pT4 $(n=4))$ was utilised to validate the relevance of methylation candidates in bladder cancer progression (Sanchez-Carbayo et al, 2006). Human bladder cancer cell lines $(n=11)$ were obtained from the American Type Culture Collection (Rockland, MD, USA) (Sanchez-Carbayo et al, 2002) and from the European Collection of Cell Cultures (Salisbury, United Kingdom). This set of cell lines included RT4, 5637, J82, T24, UM-UC-3, HT1376, HT-1197, TCCSUP, ScaBER, SW780 and RT112.

\section{CpG island arrays}

Hypermethylation patterns of genomic DNA obtained from 10 pairs of bladder tumours were profiled $v s$ their respective normal urothelium using differential methylation hybridisation as previously reported (Yan et al, 2002; Adrien et al, 2006). A panel of $12288 \mathrm{CpG}$ island clones was created from the CGI genomic library from Cross et al, 1994, as part of the Human Genome Mapping Project Resource Center (Heisler et al, 2005). Inserts from this CGI library were polymerase chain reaction (PCR)-amplified, purified by ethanol precipitation and then arrayed onto glass microscope slides by a specialised robot designed and created at Albert Einstein College of Medicine (Cheung et al, 1999; Adrien et al, 2006). The Differential Methylation Hybridisation (DMH) technique was carried out as previously described (Yan et al, 2002). Briefly, genomic DNA was initially digested with the four-base $(\mathrm{TT} \wedge \mathrm{AA})$ restriction enzyme $M s e \mathrm{I}$ at $37^{\circ} \mathrm{C}$. This digestion restricted most genomic DNA into fragments less than $200 \mathrm{bp}$ in length, and left the GC-rich $\mathrm{CpG}$ islands relatively intact. Experimental DNA fragments were ligated to linker primers using the FastLink DNA ligation kit (Epicentre, Madison, WI, USA). Samples were then digested with two methylation-sensitive restriction enzymes (Bst $\mathrm{UI}$ and HpaII) to increase coverage of the genome and to ensure complete digestion. The resulting DNA digests were purified and subsequently amplified by PCR. Using this approach, genomic DNA fragments containing unmethylated CpG sites in one sample (e.g. the reference normal urothelium specimen) were degraded by restriction digests and not be amplified. However, corresponding DNA fragments in the other sample (the bladder tumour specimen) that contain methylated restriction sites were protected from enzyme digestion and subsequently amplified by PCR. Differentially methylated sequences were identified by comparing hybridisation signals between fluorescently labelled tumour (Cy5) and reference (Cy3) amplicons when hybridised to the $\mathrm{CpG}$ microarray. Fluorescent labelling of DNA amplicons was carried out using the Klenow fragment of DNA polymerase (Fisher, Pittsburg, PA, USA) with random hexamers (Invitrogen, Carlsbad, CA, USA). Hybridisation to $\mathrm{CpG}$ arrays was carried out overnight at $50^{\circ} \mathrm{C}$ in a buffer containing $30 \%$ formamide, $3 \times$ SSC, $0.75 \%$ SDS and $100 \mathrm{ng}$ of human Cot-1 DNA. Following hybridisation, slides were briefly washed with a solution of $1 \times$ SSC, $0.1 \%$ SDS, then washed for $20 \mathrm{~min}$ at room temperature in $0.2 \times \mathrm{SSC}, 0.1 \%$ SDS and $20 \mathrm{~min}$ at room temperature in $0.1 \times$ SSC (without SDS). Slides were immediately dried as before and scanned using the GenePix 4000A microarray scanner (Molecular Devices, Sunnyvale, CA, USA).

\section{Methylation analyses of the promoter of $S O X 9$}

The CpG island methylation status of this gene was analysed by two PCR analysis strategies of bisulphite-modified genomic DNA, which induces chemical conversion of unmethylated, but not methylated, cytosine to uracil. First, methylation status was analysed by genomic sequencing of both strands of their promoters after bisulphite treatment of genomic DNA of at least eight clones per each of the bladder cancer cell lines, as previously reported ( $\mathrm{Paz}$ et al, 2003). Confirmation on at least two independent clones was required to assign methylation sites. A second strategy used methylation-specific PCR (MS-PCR) with primers specific for either the methylated or the modified unmethylated DNA. Primer sequences for bisulphite sequencing and unmethylated and methylated reactions were designed encompassing their transcription start sites distant at $723 \mathrm{bp}$ before the ATG starting codon. Table 1 summarises primers, annealing temperatures and conditions for both approaches. DNA from normal lymphocytes treated in vitro (IVD) with SssI methyltransferase was used as a positive control for methylated alleles. DNA from normal lymphocytes was used as a positive control for unmethylated alleles. PCR products were loaded onto nondenaturing $2 \%$ agarose gels, stained with ethidium bromide and visualised under an ultraviolet transilluminator.

\section{Analysis of SOX9 expression in bladder cancer cell lines}

Cell lines were treated with 1 and $5 \mu \mathrm{M}$ 5-AZA-2'-deoxycytidine (AZA; Sigma, St Louis, MO, USA) for $72 \mathrm{~h}$ to achieve demethylation (Herman and Baylin, 2003; Paz et al, 2003). RNA was isolated using RNeasy kit from Qiagen (Life Technologies, Gaithersburg, MD, USA). RNA $(1 \mu \mathrm{g})$ was reverse-transcribed using AMV Reverse Transcriptase (Promega, Madison, WI, USA) and amplified using specific primers and conditions for SOX9 (Table 1). PCR was performed using a final volume of $15 \mu \mathrm{l}$ containing $1 \times \mathrm{PCR}$ Ecostart buffer (Ecogen, Barcelona, Spain), $1.5 \mathrm{~mm}$ of $\mathrm{MgCl}_{2}$, $0.2 \mathrm{~mm}$ of dNTP, $0.25 \mu \mathrm{M}$ of each primer and $1.5 \mathrm{U}$ of Ecostart Taq polymerase (Ecogen). For PCR amplification, $0.4 \mu \mathrm{g}$ of cDNA was utilised. Reverse transcription polymerase chain reaction (RTPCR) primers were designed between different exons and encompassing large introns to avoid any amplification of genomic DNA. Glyceraldehyde-3-phosphate dehydrogenase was used as an

Table I Primer sequences and PCR conditions for bisulphite sequencing (SEQ), methylation- (MSP) and unmethylation-specific PCR (USP) and RT-PCR for SOX9

\begin{tabular}{|c|c|c|c|c|}
\hline & Sense primer $\left(5^{\prime} \rightarrow 3^{\prime}\right)$ & Antisense primer $\left(5^{\prime} \rightarrow 3^{\prime}\right)$ & $\begin{array}{l}\text { Product } \\
\text { size (bp) }\end{array}$ & $\begin{array}{l}\text { Annealing temperature } \\
\text { (PCR cycles) }\end{array}$ \\
\hline SEQ & GGGGTTGGAGAATGATITGTTAGAG & TCTAAACTAAAATСТАСССССАССТС & 434 & $62(40)$ \\
\hline MSP & GGTAGGTAGGTTCGTTITAGGC & TCTCTCCGCGACACCGAAAA & 152 & $58(34)$ \\
\hline USP & GTAGGTAGGTAGGTITGTTTTAGGT & СТСТСТССАСАAСАССАAAAАСТ & 152 & $58(34)$ \\
\hline$R T-P C R$ & AGTACCCGCACTTGCACAAC & CGTTCTTCACCGACTTCCTC & 178 & $60(25)$ \\
\hline
\end{tabular}


internal control to ensure cDNA quality and loading accuracy. The amplification products were resolved by $2 \%$ agarose gel electrophoresis and visualised by ethidium bromide staining. Cell lysates for protein analysis were analysed by western blotting using an anti-SOX-9 antibody (goat polyclonal; BD, 1/250 dilution). Equal loading was tested by re-probing with an antibody against human $\alpha$-tubulin (mouse monoclonal; Sigma, Saint Louis, MI, 1/4000 dilution). Gels were cast using the Miniprotean 3 system (Biorad, Hercules, CA, USA) and developed using ECL immunodetection reagents (Amersham Pharmacia Biotech, Piscataway, NJ, USA).

\section{Immunofluorescence}

Cells were grown on coverslips in P6 dishes, fixed in 4\% formaldehyde and fluorescently stained (Herman and Baylin, 2003; Paz et al, 2003). To monitor AZA exposure, cells were stained for SOX9 at $1 / 400$ dilution for $45 \mathrm{~min}$, using the antibody mentioned above. The secondary antibody was used at $1 / 250$ dilution. Confocal optical sections were obtained using a Leica TCS
SP microscope (Leica Microsystems, Wetzlar, Germany) equipped with krypton and argon lasers. Images were acquired and processed using the Leica LCS Lite software.

\section{Statistical analysis}

Associations among methylation patterns of SOX9 with clinicopathological variables such as tumour stage and tumour grade were evaluated using nonparametric Wilcoxon-Mann-Whitney and Kruskall-Wallis tests (Dawson-Saunders and Trapp, 1994). Associations of methylation patterns with survival were evaluated in those cases for which follow-up information were available, using the log-rank test (Dawson-Saunders and Trapp, 1994). Overall survival time was defined as the years elapsed between surgery and death from disease (or the last follow-up date). Patients who were alive at the last follow-up or lost were censored. Survival curves were plotted using Kaplan-Meier methodology. Statistical analyses were performed using the SPSS statistical package (version 8.0) (SPSS Inc., Chicago, IL, USA).

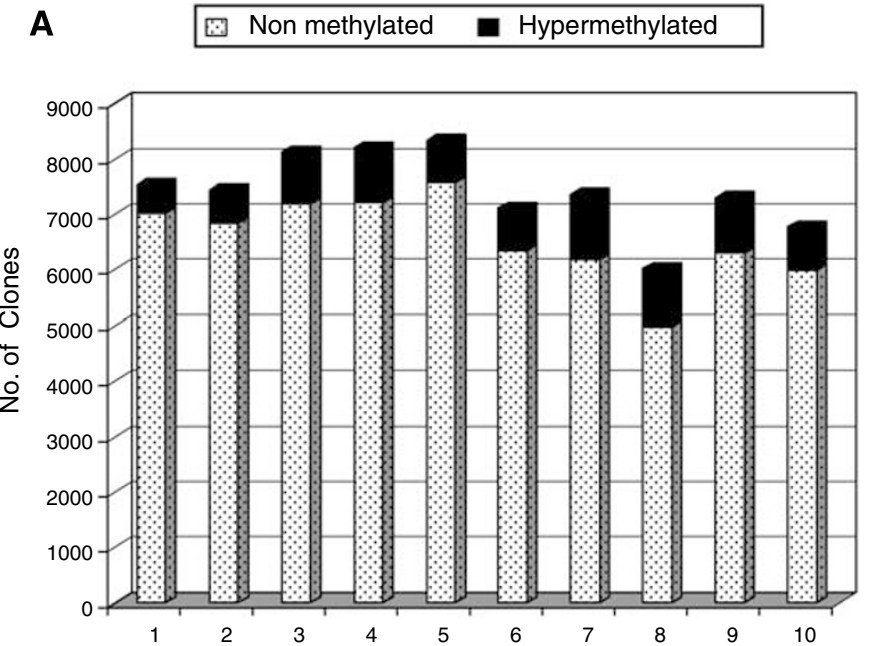

\begin{tabular}{|c|c|c|}
\hline $\begin{array}{c}\text { No. of } \\
\text { hypermethylated } \\
\text { bladder tumours }\end{array}$ & $\begin{array}{c}\text { Chromosomal } \\
\text { mapping }\end{array}$ & Gene name \\
\hline 10 & $2 \mathrm{q} 21$ & POLR2D \\
\hline 9 & 9p24 & JAK2 \\
\hline 8 & $1 q 41-q 44$ & CHMR3 \\
\hline 8 & $14 q 32.3$ & MARK3 \\
\hline 8 & $1 \mathrm{q} 32.1$ & NR5A2 \\
\hline 8 & $13 q 32.3$ & VGCNL1 \\
\hline 8 & $12 q 24$ & $R F \times 4$ \\
\hline 8 & $5 q 31.1$ & PPP2CA \\
\hline 8 & $3 q 13.3-q 21$ & $T R H$ \\
\hline 8 & $14 q 21.3$ & MAMDC1 \\
\hline 7 & $1 \mathrm{p} 12$ & GDAP2 \\
\hline 7 & $6 \mathrm{p} 23$ & $C D 83$ \\
\hline 7 & $11 \mathrm{p} 13$ & $B D N F$ \\
\hline 7 & $6 \mathrm{q} 22.1$ & LACE1 \\
\hline 7 & $13 q 12-13$ & ATP8A2 \\
\hline 7 & Xq22 & HNRPH2 \\
\hline 7 & $3 q 29$ & LRRC33 \\
\hline 7 & $2 q 37.3$ & CMKOR1 \\
\hline 7 & $9 q 31.3$ & ZNF483 \\
\hline 7 & $7 \mathrm{p} 22.3$ & VIPR2 \\
\hline 7 & $15 q 14$ & MEIS2 \\
\hline 7 & $1 \mathrm{q} 12$ & PMF1 \\
\hline 7 & $9 q 31.2$ & CTNNAL1 \\
\hline 7 & $11 q 13.5$ & INT4 \\
\hline 7 & $17 q 24.3-q 25.1$ & soxg \\
\hline 7 & $14 q 21.3$ & MAMDC1 \\
\hline
\end{tabular}

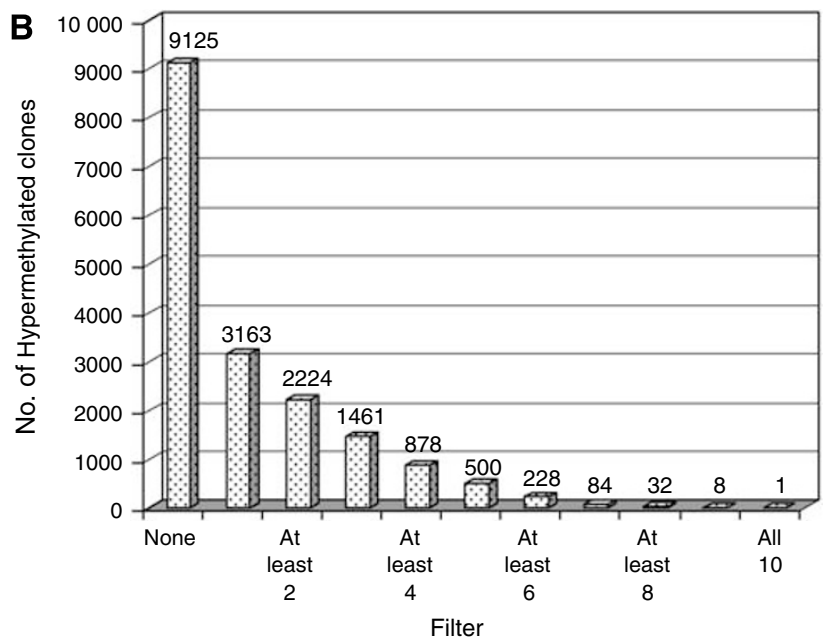

Figure I CpG arrays. (A) Quality control: the relative percentage of methylated and unmethylated genes was similar among the pairs of bladder tumours and normal urothelium samples under study. (B) CpG island arrays identify hypermethylated candidates in patients with bladder cancer. (C) Summary of known genes among the 84 clones simultaneously hypermethylated in 7 out of 10 samples under analysis. The number of cases found differentially expressed with a Cy5/Cy3 ratio higher than 2 for these genes are also indicated. 


\section{RESULTS}

Identification of methylated candidates in bladder cancer progression using $\mathrm{CpG}$ island arrays

For 10 bladder cancer patients, differential methylation hybridisation was utilised on a CpG island microarray to identify aberrantly methylated genes in advanced bladder tumour specimens (Cy5) as compared to their corresponding normal urothelium counterpart (Cy3). Among the $12288 \mathrm{CpG}$ islands analysed, half of them provided informative results. The similar rates of hypermethylated and informative clones among the 10 pairs of bladder tumours $v s$ their normal urothelium supported a similar and optimal performance of the DNA handling, labelling and hybridisation of the CpG island arrays (Figure 1A). Between 511 and 1186 clones were identified to be individually hypermethylated in each tumour sample relative to the corresponding normal urothelium tissue, based on a $\mathrm{Cy} 5 / \mathrm{Cy} 3$ ratio of 2.0 or greater (Figure $1 \mathrm{~A}$ ). Among the $12288 \mathrm{CpG}$ island clones, 84 were shown to be hypermethylated in

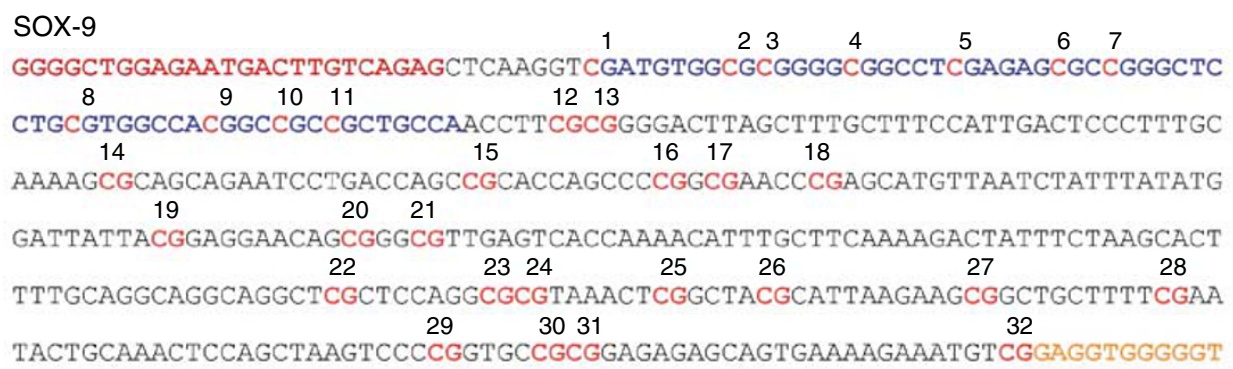

AGATCCTAGTCTAGA

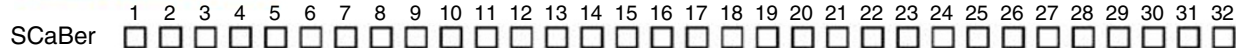

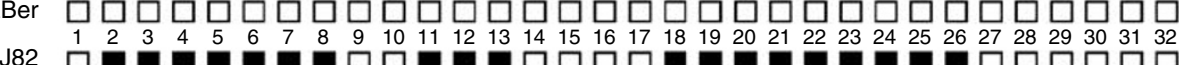
UM-UC-3

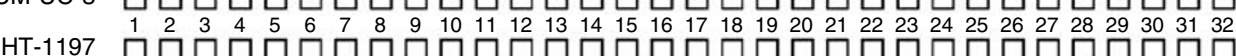

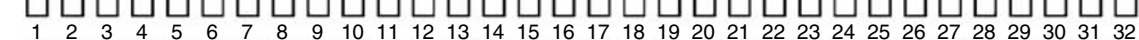

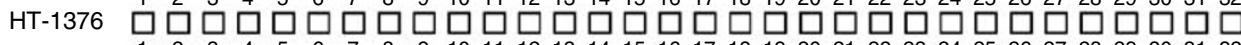

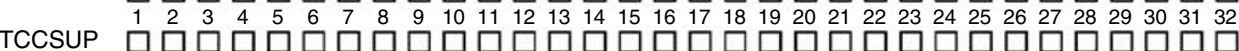

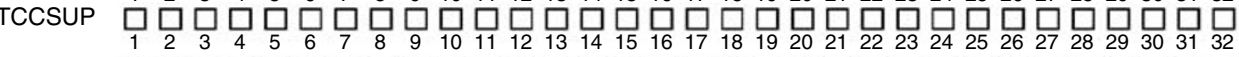

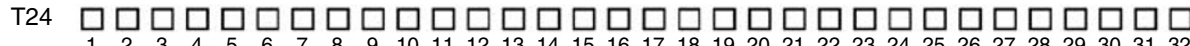

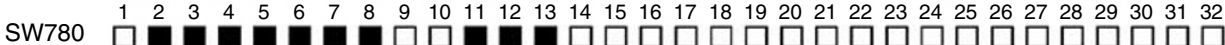

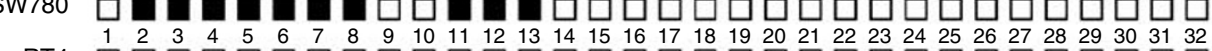

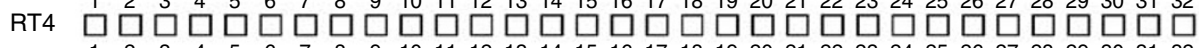

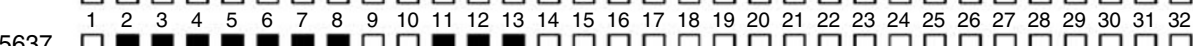

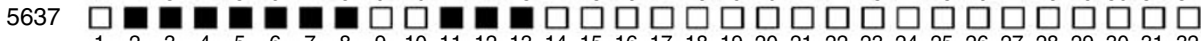

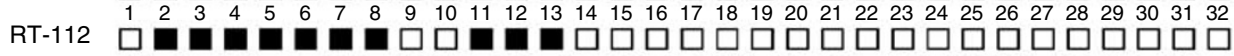
J82
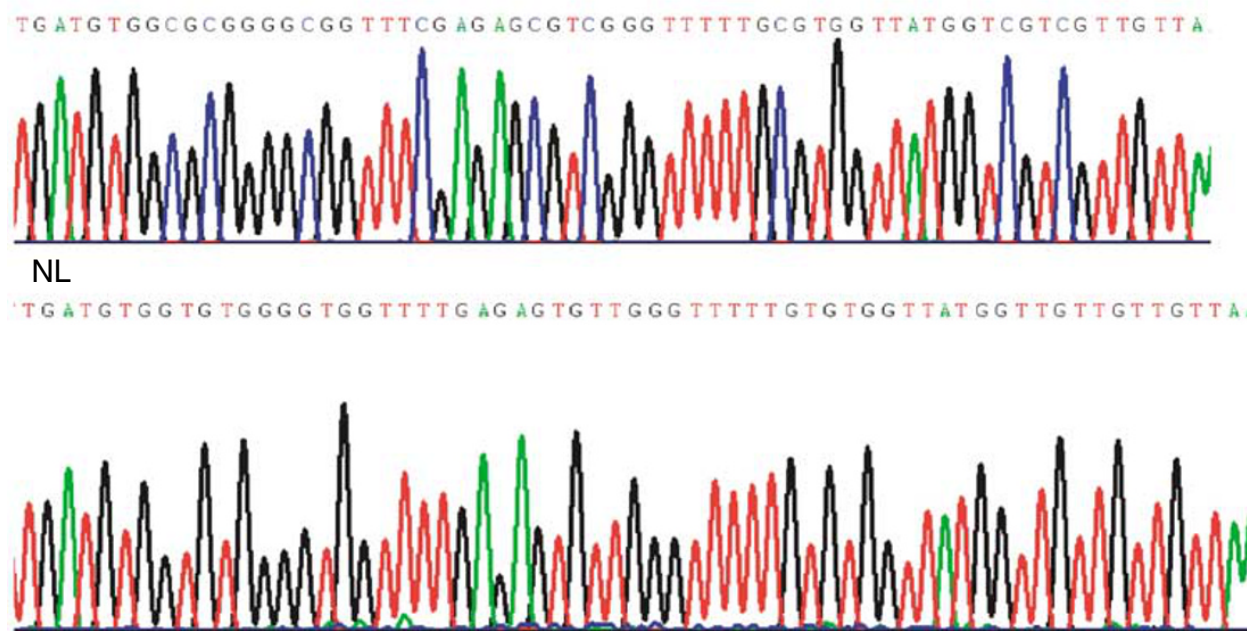

Figure 2 Analysis of CpG island methylation status of the promoter of SOX9 by bisulphite genomic sequencing in bladder human cancer cell lines ( $n=1 \mathrm{I}$; including nonmuscle invasive, invasive, metastatic transitional and squamous cells). The upper part indicates the nucleotide sequences of the CpG island region analysed by bisulphite sequencing, sequencing primers highlighted in yellow and red and the area amplified in the chromatograms in blue. The midsection shows a schematic depiction of the SOX9 CpG islands around the transcription start sites. CpG dinucleotides are represented in squares. The presence of ' $C s$ ' in the dinucleotide CpG reflects methylated cytosines (black squares), while the presence of 'Ts' in the dinucleotide CpG reflects unmethylated cytosines (white squares). Cell lines with black squares indicate the presence of methylation confirmed in at least two of the clones that were sequenced for each of the cell lines under analyses. The bottom part displays representative examples of the chromatograms obtained by bisulphite genomic sequencing of human cancer cell lines (a magnified boxed fragment is displayed). Normal lymphocytes (NL) were used as a negative sequencing control. 
$70 \%$ of the patients with invasive bladder cancer as compared to their respective normal urothelium (Figure 1B). Figure 1C summarises known genes among hypermethylated gene promoters in bladder cancer, indicating the number of cases found differentially methylated with a Cy5/Cy3 ratio higher than 2 for these genes. A complete expanded version of the 84 clones is provided as Supplementary Table 1 . Among these 84 hypermethylated clones with a $\mathrm{Cy} 5 / \mathrm{Cy} 3$ ratio higher than 2.0 in the tumour specimen of at least 7 of the 10 patients, SOX9 was selected for further validation analyses. This gene was chosen once we observed a significant statistical association of its decreasing transcript levels with increasing tumour stage $(P<0.005)$ after analysing a previously reported transcript profiling series of our group (Sanchez-Carbayo et al, 2006).

\section{SOX9 CpG island hypermethylation and its association with transcriptional gene silencing in bladder cancer cell lines}

SOX9 was tested to be a candidate gene for hypermethylationassociated inactivation in bladder cancer cells. Enriched $5^{\prime}$-CpG islands were found to be located around their transcription start sites, supporting their susceptibility to be epigenetically modified. To assess their methylation status, 11 human bladder cancer cell lines were initially screened using bisulphite genomic sequencing and MS-PCR targeted to the areas surrounding their transcription start sites. Bisulphite sequencing of these cell lines revealed $\mathrm{CpG}$ island methylation for SOX9 (Figure 2). Among the normal tissues analysed, lymphocytes (NL) and urothelium were found unmethylated at the SOX9 CpG island promoter (Figure 2).

Having observed promoter hypermethylation in bladder cancer cell lines by bisulphite sequencing, specific MS-PCR was performed for SOX9 (Figure 3). Methylation patterns observed by bisulphite sequencing highly correlated with the results obtained by MS-PCR, with differences attributable to the use of primers in these techniques encompassing slightly different areas around the transcription start sites for both strategies (Figure 2). Methylation analyses were then linked to transcript and protein expression estimates of the gene under study. The association between these epigenetic aberrations and putative transcriptional inactivation of this gene was initially assessed at the RNA and protein levels. Bladder cancer cell lines hypermethylated for SOX 9 showed low transcript and protein expression as revealed by RT - PCR and western blot analyses (Figure 3).

Treatment of methylated and unmethylated bladder cancer cell lines with a DNA-demethylating agent served to further link SOX9 hypermethylation and gene silencing. Exposure of methylated bladder cancer cell lines to the demethylating drug, AZA, restored expression of SOX9 at the transcript level in the J82 cell line. RT4 was used as the control cell line to assess the specificity of AZA exposure, but not to modify gene expression of this candidate gene in unmethylated bladder cancer cells (Figure 4). Western blot and immunofluorescence analyses were performed to confirm that protein expression was also restored after AZA exposure. Overall, the results indicated a high correlation of methylation data with gene expression, observations especially supported by AZA reactivation analyses.

\section{SOX9 is frequently hypermethylated in primary bladder tumours, and associated with clinicopathological variables}

Once the functional consequences of SOX9 CpG island hypermethylation were determined in vitro, it was tested whether hypermethylation of this gene was cancer-specific. Comparison of methylation of bladder tumours and their respective pairs of normal urothelium was analysed on an independent set of 10 cases for which paired normal urothelium DNA was available. Methylation was found in $70 \%$ of the bladder tumours and $10 \%$ of the normal urothelium tested. Illustrative examples of MS-PCR results among bladder tumours and paired normal urothelium are shown in Figure 5A. The relevance of their methylation in human clinical material was further analysed by MS-PCR in an independent large set of 101 primary bladder tumours. Hypermethylation patterns of this gene among these samples can be assessed in Figure 5B. Overall, SOX9 CpG island hypermethylation was respectively found in $56.4 \%$ of the cases (57/101). The next analyses dealt with evaluating the link between the hypermethylation status of SOX9 and clinicopathological variables of bladder cancer patients, as summarised in Figure 5B. Overall, tumours displaying high grade were more frequently methylated than those with low grade. A significant association of hypermethylation with tumour grade was found for SOX9 (Mann-Whitney, $P=0.032$ ). Interestingly, SOX9 hypermethylation was significantly associated with shorter overall survival (log-rank, $P=0.025 ;$ Figure $5 \mathrm{C}$ ). Therefore, SOX9
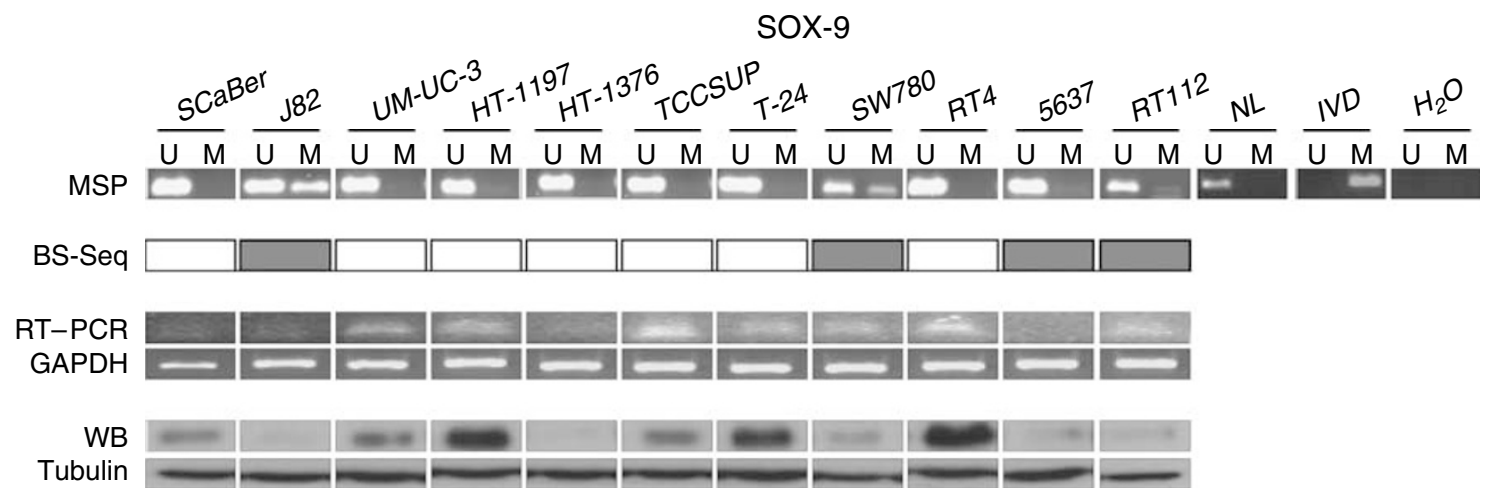

Figure 3 CpG island methylation is associated with gene silencing of SOX9. The upper part shows methylation-specific PCRs for SOX9 in human bladder cancer cell lines. The presence of a PCR band under the lane $M$ indicates a methylated gene, while the presence of a PCR band under the lane $U$ indicates an unmethylated gene. Normal lymphocytes (NL) and in vitro-methylated DNA (IVD) were used as negative and positive controls for unmethylated and methylated PCRs, respectively. Sequencing information is included as well, highlighting methylated cell lines by genome sequencing in dark grey. Reverse transcription polymerase chain reaction analysis of SOX9 expression is displayed. Glyceraldehyde-3-phosphate dehydrogenase (GAPDH) transcript expression was used as a transcript loading control. Western blot analysis of protein expression is also shown. Tubulin expression was used as a protein loading control. The hypermethylated cell lines show relatively low transcript and protein expression of the coded protein as compared to unmethylated cell lines. 

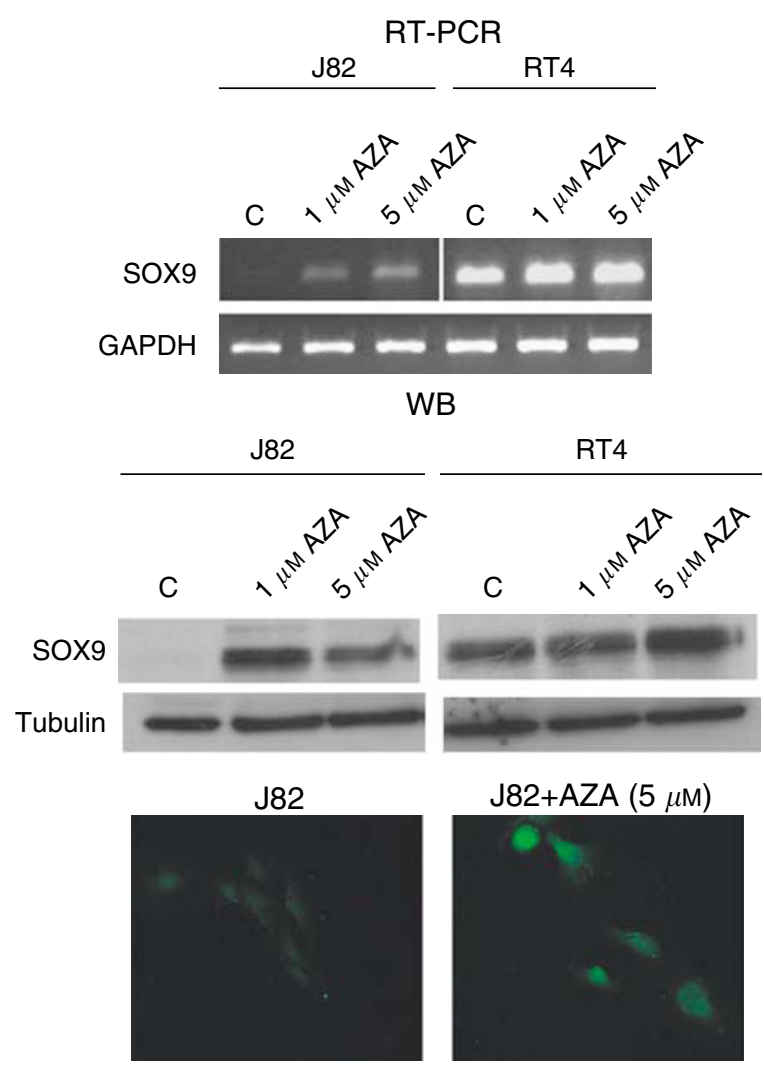

RT4
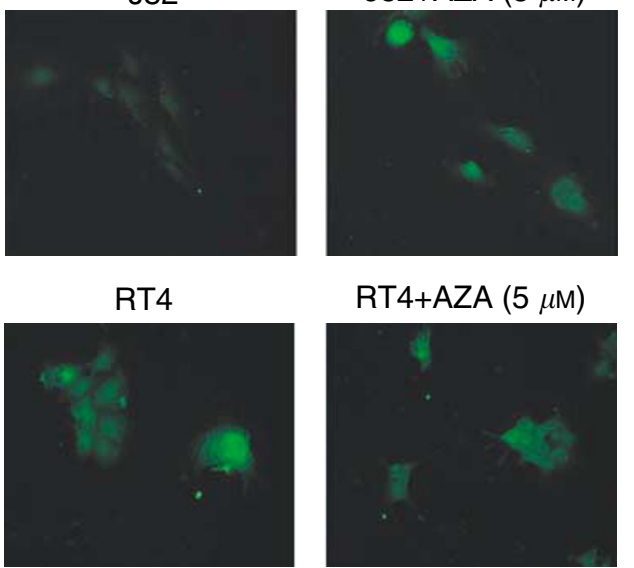

RT4+AZA (5 $\mu \mathrm{M})$

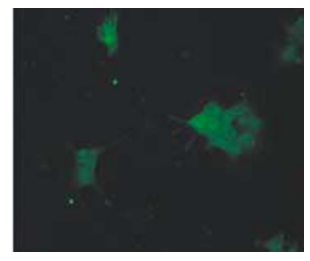

Figure 4 The treatment with the demethylating agent AZA reactivates gene expression of SOX9. The upper part displays the reverse transcription polymerase chain reaction analysis of SOX9 expression. Glyceraldehyde-3-phosphate dehydrogenase (GAPDH) expression was used as a transcript loading control. The hypermethylated 82 cell line did not express SOX9, and restored SOX9 transcript expression after AZA exposure. The mid-section shows western blot analysis of protein expression. Tubulin expression was used as a protein loading control. The hypermethylated cell line did not express the coded protein. The treatment with the demethylating agent reactivated SOX9 protein expression. The unmethylated RT4 cell line did not show changes in transcript or protein expression. The bottom part displays immunofluorescence analysis of SOX9 expression after AZA exposure. The methylated cell line did not show any staining for the protein, while the unmethylated ones showed its characteristic staining pattern.

hypermethylation was identified to be a likely predictor of poor outcome.

\section{DISCUSSION}

The use of high-throughput profiling approaches is accelerating the discovery of genetic and epigenetic events associated with tumorigenesis and tumour progression. This study represents the first report describing the use of $\mathrm{CpG}$ island arrays as a means to comprehensively identify hypermethylation candidates in bladder cancer. By using a comprehensive approach, hypermethylation of novel genes was identified along bladder cancer progression due to the strategy of comparing bladder tumours and normal urothelium counterparts. Among the genes showing twofold hypermethylation in at least 7 of 10 cancers, SOX9 gene was chosen for further analysis. To our knowledge, there was no data suggesting that SOX9 could be epigenetically modified by methylation in human cancer. This gene was chosen once we observed significant statistical associations of its decreasing transcript levels with increasing tumour stage $(P<0.005)$ after analysing a previously reported transcript profiling data of our group (Sanchez-Carbayo et al, 2006), suggesting their involvement in bladder cancer progression. This clinical observation excluded the need to estimate multiple testing and false discovery thresholds for SOX9, a common strategy undertaken for candidate selection in high-throughput studies.

The consequences of $\mathrm{CpG}$ island hypermethylation of candidate genes in bladder cancer progression need to be assessed from the standpoint of mechanistic, biological and translational implications. Mechanistically, it is important to evaluate the cellular consequences of the methylation of the promoter of SOX9 in bladder cancer cells. Several techniques were utilised to link methylation analyses with expression estimates of the gene under study. Methylation status and expression results of SOX9 correlated to high extent among a variety of bladder cell lines representing the spectrum of bladder cancer progression in vitro. AZA exposure experiments confirmed the impact of methylation in the expression of this gene by specifically restoring their transcript and protein expression in methylated bladder cancer cells. Overall, in vitro analyses demonstrated that the expression of SOX9 is aberrantly silenced by CpG island promoter hypermethylation in bladder cancer, observations especially supported by AZA reactivation analyses.

To the best of our knowledge, SOX9, the gene identified in the present report, had not been previously related to bladder cancer. SOX9 is a transcription factor that is expressed in chondrocytes and several tissues, including the central nervous and urogenital systems (Huang et al, 2000; Soderstrom et al, 2002). SOX9 has been shown to be relevant at distinguishing mesenchymal chondrosarcoma from other small blue round cell tumours (Soderstrom et al, 2002; Wehrli et al, 2003) and at modulating retinoid-mediated growth in breast cancer cells (Afonja et al, 2002). Growth and tumorigenicity suppression associated with SOX9 has been found in prostate, breast and colon cells (Afonja et al, 2002; Drivdahl et al, 2004; Jay et al, 2005). The epigenetic silencing of SOX9 may aid understanding as to how it contributes to tumorigenesis and tumour progression in such types of neoplasias. Future studies are also warranted to dissect such mechanisms in the context of bladder cancer.

More importantly, the translational implications of the discovery of the methylation of this novel candidate gene identified by the CpG array have also been addressed in this work. CpG arrays identified higher methylation rates of the potential methylation candidate genes in tumour specimens as compared to normal urothelium counterparts. It was then necessary to test whether SOX9 methylation was a cancer-specific epigenetic event. An independent set of 10 pairs of bladder tumours and normal urothelium, similar to the set used for the discovery of explorative analyses, was utilised to validate the presence of cancer-specific methylation of SOX9 by an independent method, MS-PCR. SOX 9 methylation was found with rates higher than $70 \%$ in bladder tumours and lower than $20 \%$ in their normal urothelium counterparts, an observation suggesting that SOX9 could be methylated in a cancer-specific manner. Methylation in normal urothelium by MS-PCR could be attributed to the presence of mixed subpopulations to a certain extent detectable by a PCRbased technique, or that methylation could be a very early cancer epigenetic event, especially detectable in phenotypically normal urothelium counterparts due to the field effect. The initial 


\begin{tabular}{|c|c|c|}
\hline & \multicolumn{2}{|c|}{ SOX9 } \\
\hline STAGE & $\mathrm{U}(44)$ & $\mathrm{M}(57)$ \\
\hline pTa (24) & 12 & 12 \\
\hline pT1 (32) & 13 & 19 \\
\hline pT2 (16) & 6 & 10 \\
\hline pT3 (25) & 13 & 12 \\
\hline pT4 (4) & 0 & 4 \\
\hline GRADE & $\mathrm{U}(44)$ & $\mathrm{M}(57)$ \\
\hline G1 (5) & 5 & 0 \\
\hline G2 (15) & 7 & 8 \\
\hline G3 (81) & 32 & 49 \\
\hline
\end{tabular}

C

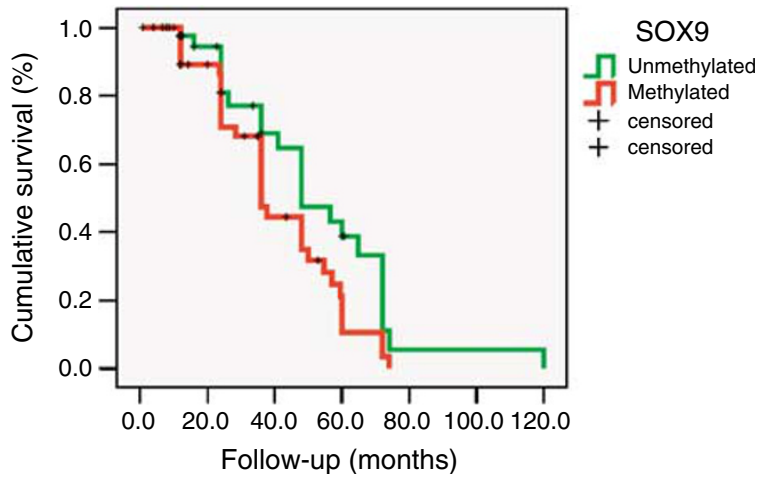

Figure 5 Association between SOX9 hypermethylation with cancer progression and clinical outcome in bladder tumours. (A) Representative pairs of normal urothelium (NB) and primary bladder tumours (T) analysed by MS-PCR for SOX9. The presence of a PCR band under the lane M indicates a methylated gene, while the presence of a PCR band under the lane $U$ indicates an unmethylated gene. Normal lymphocytes (NL) and in vitro-methylated DNA (IVD) are used as negative and positive controls for unmethylated and methylated PCRs, respectively. (B) Summary of the number of unmethylated $(\cup)$ and methylated $(M)$ cases for SOX9 regarding their tumour stage and tumour grade. $(\mathbf{C})$ Kaplan-Meier curve describing the association of SOX9 hypermethylation with poor overall survival.

discovery of this methylated candidate gene by comparing bladder tumours and their respective normal urothelium counterparts on the CpG arrays together with these observations on an independent set of cases were consistent with the concept of cancerspecific hypermethylation. This is a critical issue that requires to be tested for any hypermethylated candidates since the CpG arrays identified methylation higher in tumour specimens than in normal counterparts, without necessarily implying this epigenetic event to be cancer-specific.

It should be mentioned that several qualitative and quantitative methodologies are available for the purpose of determining methylation status of tumour specimens (Esteller, 2007). We selected standard gel-based MS-PCR, which could be considered appropriate enough for our descriptive analyses aiming at distinguishing between methylated and unmethylated cases. Considering the issue of tissue heterogeneity, only the complete absence of a PCR band was reported as a negative result. Quantitative RT-PCR methodologies are known to show their main advantages for comparative quantitative methylation analyses especially in body fluids. MS-PCR analyses in bladder cancer cell lines and independent sets of bladder tumours showed highly varying hypermethylation rates. CpG arrays and MS-PCR in the discovery and validation sets of 10 pairs of tumours and normal urothelium revealed the presence of SOX9 methylation in at least $70 \%$ of the invasive cases analysed. Methylation rates were closer to $60 \%$ in the larger series of bladder tumours, which confirmed that SOX 9 hypermethylation is a frequent event in bladder cancer. Moreover, this independent set of 101 bladder tumours served to explore the clinical relevance of the identified methylation for SOX9.

The association of SOX9 hypermethylation with bladder cancer progression can be justified as follows: first, the experimental design of the discovery of this methylated candidate gene by comparing invasive tumours $v s$ their respective normal urothelium associates it with bladder cancer progression, providing cancer specificity to this candidate confirmed using independent sets of pairs of tumours and normal urothelium; second, this gene was selected based on its statistical association with tumour stage using transcript profiling analyses of bladder tumours $(P<0.005)$; third, it was shown that methylation of $S O X 9$ is a frequent event among bladder tumours using an independent larger series of patients with bladder cancer. The methylation rates of $55.3 \%$ of the nonmuscle invasive cases showed that this epigenetic modification is an early event in bladder cancer. Moreover, the significant association of SOX9 methylation with clinicopathological tumour grade and survival further supported their association with cancer progression. Interestingly, the presence of SOX 9 hypermethylation was associated with overall survival, confirming its potential role as a prognostic marker in the clinical management of patients affected with uroepithelial tumours. Such finding meets concordance with the knowledge of the involvement of this gene in human solid neoplasias. Inactivation by hypermethylation of SOX9, a gene reported to be a potential tumour suppressor, would be justified to be a poor outcome prognosticator (Afonja et al, 2002; Wehrli et al, 2003; Drivdahl et al, 2004). Thus, the gene identified in the present work represents a novel methylated tumour suppressor candidate to be investigated in bladder cancer. In addition to clinicopathological stratification of patients with bladder cancer, a relevant translational point relates to treatment, since it poses the potential use of demethylating agents such as 5azacytidine in bladder cancer, to reactivate CpG island-hypermethylated genes, as has been approved by the Food and Drug Administration for a preleukemic disease, the myelodysplastic syndrome (Esteller, 2007). In this new scenario, SOX9 represents a candidate target gene supporting the potential use of this type of drugs to achieve their demethylation and reactivation in bladder tumours. Further research would identify target-specific demethylating agents due to the complexity of epigenetic events along tumour progression.

In summary, our study discovers novel hypermethylated candidate genes in bladder cancer. It provides a mechanistic explanation for the observed loss of SOX9 in uroepithelial malignancies, demonstrating transcriptional silencing by promoter CpG island hypermethylation in bladder cancer cell lines. Although the ultimate consequences of its epigenetic inactivation in the molecular biology of the tumour cell remain unknown, its hypermethylation emerges as a strong indicator of tumour progression and clinical outcome for bladder cancer patients. These data should encourage further research into its downstream biological impact in tumorigenesis and cancer progression and 
eventually as a potential therapeutic target to be addressed following an epigenetic approach.

\section{ACKNOWLEDGEMENTS}

We would like to thank all the members of the laboratories of Drs Belbin, Cordon- Cardo, Esteller and Sánchez-Carbayo for their technical support and constructive suggestions in the preparation

\section{REFERENCES}

Adrien LR, Schlecht NF, Kawachi N, Smith RV, Brandwein-Gensler M, Massimi A, Chen S, Prystowsky MB, Childs G, Belbin TJ (2006) Classification of DNA methylation patterns in tumor cell genomes using a CpG island microarray. Cytogenet Genome Res 114: 16-23

Afonja O, Raaka BM, Huang A, Das S, Zhao X, Helmer E, Juste D, Samuels HH (2002) RAR agonists stimulate SOX9 gene expression in breast cancer cell lines: evidence for a role in retinoid-mediated growth inhibition. Oncogene 21: $7850-7860$

Catto JW, Azzouzi AR, Rehman I, Feeley KM, Cross SS, Amira N, Fromont G, Sibony M, Cussenot O, Meuth M, Hamdy FC (2005) Promoter hypermethylation is associated with tumor location, stage, and subsequent progression in transitional cell carcinoma. J Clin Oncol 23: $2903-2910$

Chapman EJ, Harnden P, Chambers P, Johnston C, Knowles MA (2005) Comprehensive analysis of CDKN2A status in microdissected urothelial cell carcinoma reveals potential haploinsufficiency, a high frequency of homozygous co-deletion and associations with clinical phenotype. Clin Cancer Res 11: $5740-5747$

Cheung VG, Morley M, Aguilar F, Massimi A, Kucherlapati R, Childs G (1999) Making and reading microarrays. Nat Genet 21: 15-19

Ching TT, Maunakea AK, Jun P, Hong C, Zardo G, Pinkel D, Albertson DG, Fridlyand J, Mao JH, Shchors K, Weiss WA, Costello JF (2005) Epigenome analyses using BAC microarrays identify evolutionary conservation of tissue-specific methylation of SHANK3. Nat Genet 37: $645-651$

Cordon-Cardo C, Cote RJ, Sauter G (2000) Genetic and molecular markers of urothelial premalignancy and malignancy. Scand J Urol Nephrol Suppl 205: $82-93$

Costello JF, Fruhwald MC, Smiraglia DJ (2000) Aberrant CpG-island methylation has non-random and tumour-type-specific patterns. Nat Genet 24: $132-138$

Cross SH, Charlton JA, Nan X, Bird AP (1994) Purification of CpG islands using a methylated DNA binding column. Nat Genet 6: 236-244

Dawson-Saunders B, Trapp RG (1994) Basic \& Clinical Biostatistics 2nd edn, Norwalk, Connecticut: Appleton \& Lange

Drivdahl R, Haugk KH, Sprenger CC, Nelson PS, Tennant MK, Plymate SR (2004) Suppression of growth and tumorigenicity in the prostate tumor cell line M12 by overexpression of the transcription factor SOX9. Oncogene 23: 4584-4593

Esteller M (2002) CpG island hypermethylation and tumor suppressor genes: a booming present, a brighter future. Oncogene 21: 5427-5440

Esteller M (2007) Cancer epigenomics: DNA methylomes and histonemodification maps. Nat Rev Genet 8: 286-298

Esteller M, Corn PG, Baylin SB (2001) A gene hypermethylation profile of human cancer. Cancer Res 61: 3225-3229

Feinberg AP, Ohlsson R, Henikoff S (2006) The epigenetic progenitor origin of human cancer. Nat Rev Genet 7: 21-33

Heisler LE, Torti D, Boutros PC, Watson J, Chan C, Winegarden N, Takahashi M, Yau P, Huang TH, Farnham PJ, Jurisica I, Woodgett JR, Bremner R, Penn LZ, Der SD (2005) CpG island microarray probe sequences derived from a physical library are representative of CpG Islands annotated on the human genome. Nucleic Acids Res 33: $2952-2961$

Herman JG, Baylin SB (2003) Gene silencing in cancer in association with promoter hypermethylation. N Engl J Med 349: $2042-2054$

Huang W, Zhou X, Lefebvre V, de Crombrugghe B (2000) Phosphorylation of SOX9 by cyclic AMP-dependent protein kinase A enhances SOX9's ability to transactivate a Col2a1 chondrocyte-specific enhancer. Mol Cell Biol 20: $4149-4158$ of this manuscript. We would like to thank the Tissue Procurement Core, especially Cora Mariano, at Memorial Sloan-Kettering Cancer Center for their support in facilitating the tumour specimens and clinical follow-up of the bladder cancer cases analysed in this study.

Supplementary Information accompanies the paper on British Journal of Cancer website (http://www.nature.com/bjc)

Jay P, Berta P, Blache P (2005) Expression of the carcinoembryonic antigen gene is inhibited by SOX9 in human colon carcinoma cells. Cancer Res 65: $2193-2198$

Jones PA, Baylin SB (2002) The fundamental role of epigenetic events in cancer. Nat Rev Genet 3: 415-428

Kim WJ, Kim EJ, Jeong P, Quan C, Kim J, Li QL, Yang JO, Ito Y, Bae SC (2005) RUNX3 inactivation by point mutations and aberrant DNA methylation in bladder tumors. Cancer Res 65: $9347-9354$

Lee MG, Kim HY, Byun DS (2001) Frequent epigenetic inactivation of RASSF1A in human bladder carcinoma. Cancer Res 61: 6688-6692

Liang G, Gonzales FA, Jones PA, Orntoft TF, Thykjaer T (2002) Analysis of gene induction in human fibroblasts and bladder cancer cells exposed to the methylation inhibitor 5-aza-2'-deoxycytidine. Cancer Res 62: 961-966

Markl ID, Cheng J, Liang G (2001) Global and gene-specific epigenetic patterns in human bladder cancer genomes are relatively stable in vivo and in vitro over time. Cancer Res 61: $5875-5884$

Marsit CJ, Karagas MR, Andrew A, Liu M, Danaee H, Schned AR, Nelson $\mathrm{HH}$, Kelsey KT (2005) Epigenetic inactivation of SFRP genes and TP53 alteration act jointly as markers of invasive bladder cancer. Cancer Res 65: $7081-7085$

Muto S, Horie S, Takahashi S (2000) Genetic and epigenetic alterations in normal bladder epithelium in patients with metachronous bladder cancer. Cancer Res 60: 4021-4025

Paz MF, Fraga MF, Avila S, Guo M, Pollan M, Herman JG, Esteller M (2003) A systematic profile of DNA methylation in human cancer cell lines. Cancer Res 63: 1114-1121

Sanchez-Carbayo M, Socci ND, Charytonowicz E, Lu M, Prystowsky M, Childs G, Cordon-Cardo C (2002) Molecular profiling of bladder cancer using cDNA microarrays: defining histogenesis and biological phenotypes. Cancer Res 62: 6973-6980

Sanchez-Carbayo M, Socci ND, Lozano J, Saint F, Cordon-Cardo C (2006) Defining molecular profiles of poor outcome in patients with invasive bladder cancer using oligonucleotide microarrays. J Clin Oncol 24: 778-789

Soderstrom M, Bohling T, Ekfors T, Nelimarkka L, Aro HT, Vuorio E (2002) Molecular profiling of human chondrosarcomas for matrix production and cancer markers. Int J Cancer 100: 144-151

Stoehr R, Wissmann C, Suzuki H, Knuechel R, Krieg RC, Klopocki E, Dahl E, Wild P, Blaszyk H, Sauter G, Simon R, Schmitt R, Zaak D, Hofstaedter F, Rosenthal A, Baylin SB, Pilarsky C, Hartmann A (2004) Deletions of chromosome $8 \mathrm{p}$ and loss of sFRP1 expression are progression markers of papillary bladder cancer. Lab Invest 84: $465-478$

Urakami S, Shiina H, Enokida H, Kawakami T, Tokizane T, Ogishima T, Tanaka Y, Li LC, Ribeiro-Filho LA, Terashima M, Kikuno N, Adachi H, Yoneda T, Kishi H, Shigeno K, Konety BR, Igawa M, Dahiya R (2006) Epigenetic inactivation of Wnt inhibitory factor-1 plays an important role in bladder cancer through Aberrant Canonical Wnt/ß-Catenin signaling pathway. Clin Cancer Res 12: 383-391

Wehrli BM, Huang W, De Crombrugghe B, Ayala AG, Czerniak B (2003) Sox9, a master regulator of chondrogenesis, distinguishes mesenchymal chondrosarcoma from other small blue round cell tumors. Hum Pathol 34: $263-269$

Wolff EM, Liang G, Jones PA (2005) Mechanisms of disease: genetic and epigenetic alterations that drive bladder cancer. Nat Clin Pract Urol 2: $502-510$

Yan PS, Perry MR, Laux DE, Asare AL, Caldwell CW, Huang TH (2000) CpG island arrays: an application toward deciphering epigenetic signatures of breast cancer. Clin Cancer Res 6: 1432 - 1438

Yan PS, Wei SH, Huang TH (2002) Differential methylation hybridization using CpG island arrays. Methods Mol Biol 200: 87-100 